\title{
An Optimal Design Strategy for a Thomson Coil Actuator
}

\author{
Dong-Kuk Lim*, Sang-Yong Jung**, Hyun-Kyo Jung* and Jong-Suk Ro ${ }^{\dagger}$
}

\begin{abstract}
An arc eliminator allows a surge current bypass rapidly into the earth to facilitate the circuit breaking process and increase the stability of the load circuit. Thomson coil actuator (TCA) is a type of actuator that can function as an arc eliminator. The TCA has a simple structure and significantly rapid speed compared to the other types of actuators. In this paper, significant variables, which have a dominant effect on the performance of the TCA, are investigated in detail. Using these variables and an optimization algorithm, an optimal design strategy for the TCA is proposed in this research. The efficacy of the proposed optimal design strategy and the feasibility of the application of the designed TCA for a high power circuit breaker as the arc eliminators are validated through the experiment.
\end{abstract}

Keywords: Actuator, Circuit breaker, Optimization algorithm, Thomson coil actuator

\section{Introduction}

When a surge current flows into a load circuit while circuit breaking, the surge current has to be eliminated as soon as possible to minimize the damage of the load circuit. The surge current rapidly bypasses the arc eliminator into the ground just before activating the circuit breaker (CB) to minimize the arc while circuit breaking, mitigating the load of the circuit breaker for the protection of the load circuit. Hence, the arc eliminator can increase the stability and reliability of the circuit breaker through its faster operation than the circuit breaker and can be useful for the application of a high power CB [1-3]. Furthermore, the arc eliminator can be useful to a high power application because the arc in the high power application is significant.

These merits have encouraged many researchers to study arc eliminators for several decades. Numerous types of actuators such as spring actuators, electromagnetic actuators, linear motors, and Thomson coil actuators (TCA) are proposed for the arc eliminator [4-6]. Especially, the TCA has received much attention due to its rapid completion time in several milliseconds [6-9]. Most research on the TCA focus on the basic working principles or on the characteristic analysis of the TCA. The trial and error method has been widely used for the design of the TCA. The TCA should be analyzed by using the time consuming finite element method (FEM) with time difference method (TDM). Hence, it takes a lot of time to design the TCA.

To solve this conventional problem, we proposed an optimal design strategy applying an optimization algorithm in this manuscript. Specifically, an analysis method which

$\dagger$ Corresponding Author: School of Electrical and Electronics Engineering, ChungAng University, Korea. (jongsukro@naver.com)

* Dept. of Electrical and Computer Engineering, Seoul National University, Korea. (ldk8745@gmail.com, hkjung@snu.ac.kr)

** School of Electronic and Electrical Engineering, Sungkyunkwan University, Korea. (syjung@skku.edu)

Received: April 8, 2016; Accepted: July 25, 2016 uses time difference method is derived in this paper.

Furthermore, variables of the TCA and its effect on the performance of the TCA are investigated in detail. An optimal design strategy incorporating significant design variables of the TCA is proposed by applying a deterministic optimization method in this research.

The usefulness of the optimal design strategy and the

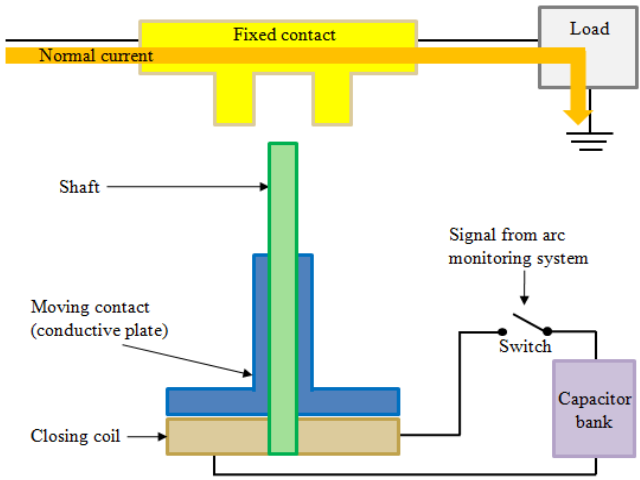

(a)

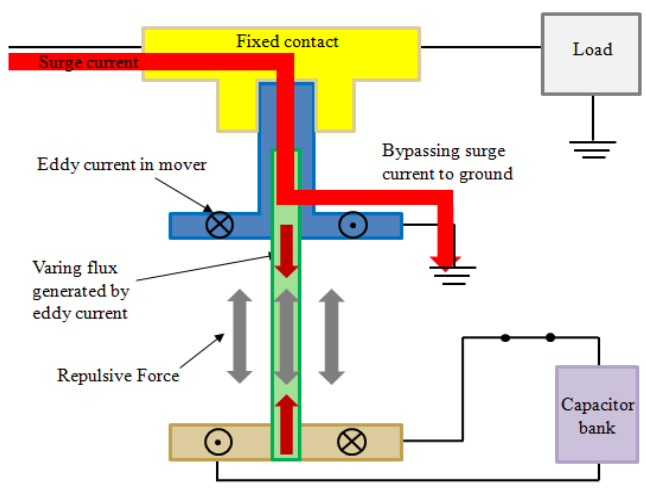

(b)

Fig. 1. (a) Normal state of the TCA: (b) Operation of the TCA to bypass the surge current 
feasibility of the application of the designed TCA for the arc eliminator are verified through the experiment.

\section{Structure and Working Principle of the TCA}

As shown in Fig. 1(a), the TCA consists of a mover, a closing coil, and a capacitor bank. At the initial stage, the mover is an open state and the capacitor is charged. If the arc monitoring system senses the surge current, the closing coil is excited by the current from the charged capacitor.

As the current in the closing coil increases, a variable magnetic flux is generated. This variable magnetic flux induces an eddy current in the mover. Hence, a repulsive force is generated by the magnetic flux of the mover and the closing coil, as shown in Fig. 1(b). The mover is moved by the repulsive force between the mover and the closing coil. Finally, the mover, which is a moving electrode, comes into contact with the fixed contactor and the surge current can bypass to the ground.

\section{Analysis of the TCA by using TDM}

The commercial FEM analysis tool, JMAG, is used in this research for the analysis of the TCA. Governing equations for the analysis of the TCA are derived as follows:

Fig. 2 shows the equivalent circuit of the TCA $\left(V_{S}\right.$ : the source voltage, $C$ : the capacitance of the capacitor, $V_{C}$ : the charged voltage at the capacitor, $L_{\text {coil }}$ : is the self-inductance of the closing coil, $L_{\text {mover }}$ : the self-inductance of the mover, $M$ : the mutual-inductance, $R_{\text {coil }}$ : the resistance of the closing coil, $R_{\text {mover }}$ : the resistance of the mover, and $S_{i}$ : the $i^{\text {th }}$ switch).

Before the closing operation, $S_{1}$ is turned on and the capacitor is charged by the DC voltage source. When the arc monitoring system senses the surge current, the closing coil is excited by the current from the charged capacitor by turning the switch $S_{1}$ off and the switch $S_{2}$ on.

Eqs. (1)-(3) represent the voltage equation applied the TDM for the closing coil and the mover.

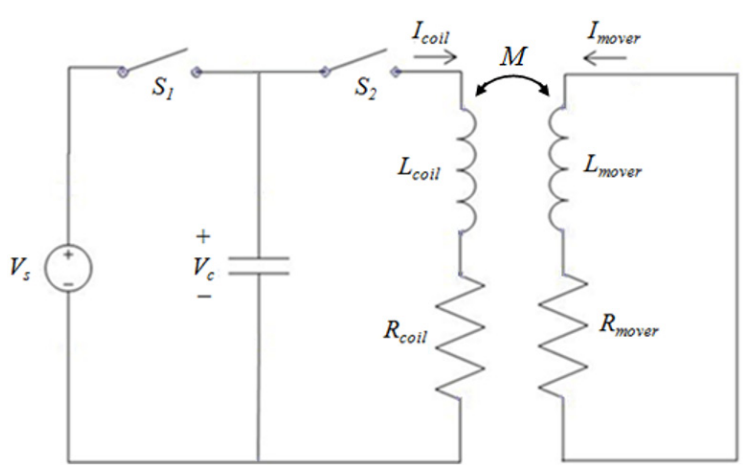

Fig. 2. The equivalent circuit of the TCA

$$
\begin{gathered}
V_{c}^{n}=\left(I_{\text {coil }}{ }^{n-1}+d I_{\text {coil }}{ }^{n}\right) R_{\text {coil }}+L_{\text {coil }}{ }^{n-1} \frac{d I_{\text {coil }}{ }^{n}}{d t} \\
-M^{n-1} \frac{d I_{\text {mover }}{ }^{n}}{d t}-\frac{d M^{n-1}}{d x} \frac{d x}{d t} I_{\text {mover }}{ }^{n} \\
0=\left(I_{\text {mover }}{ }^{n-1} d I_{\text {mover }}{ }^{n}\right) R_{\text {mover }}+L_{\text {mover }}{ }^{n-1} \frac{d I_{\text {mover }}{ }^{n}}{d t} \\
-M^{n-1} \frac{d I_{\text {coil }}{ }^{n}}{d t}-\frac{d M^{n-1}}{d x} \frac{d x}{d t} I_{\text {coil }}{ }^{n} \\
V_{c}^{n}=V_{c}^{n-1}+\frac{1}{C}\left(I_{\text {coil }}{ }^{n-1}+d I_{\text {coil }}{ }^{n}\right) d t
\end{gathered}
$$

where $d I_{\text {mover }}$ and $d I_{\text {coil }}$ are the change of the current in the mover and the coil; $d x, d t$, and $d M$ are the change of the displacement, the time, and the mutual inductance; $n$ is the $n^{\text {th }}$ time step [9-12].

By analyzing the equation of motion, the displacement and the velocity of the mover can be calculated. The velocity of the mover $v$ is derived through the integration of the acceleration of the mover $a$ by (4) and the displacement of the mover $x$ is induced by $(5)$. ( $m$ : the mass of the mover, $g$ : the acceleration of the gravity, $F_{\text {electro }}$ : the electromagnetic force acting on the mover calculated by the FEM, and $F_{f r i c}$ : the frictional force)

$$
\begin{aligned}
& v^{n}=v^{n-1}+d v^{n}=v^{n-1}+\left(\frac{F_{\text {electro }}^{n}+F_{\text {fric }}^{n}-m g}{m}\right) d t \\
& x^{n}=x^{n-1}+v^{n-1} d t+\frac{1}{2} a^{n} d t^{2}
\end{aligned}
$$

The commercial FEM analysis tool, JMAG, takes into account the skin effect. The skin effect is the tendency of alternating current to become distributed within a conductor such that the current density is largest near the surface of the conductor. The current flows mainly at the skin of the conductor, between the outer surface and a lever called the skin depth $\delta$ and it is computed as

$$
\delta=\frac{1}{\sqrt{\sigma \mu f \pi}}
$$

where $\sigma$ is the conductivity of conductor, $\mu$ is permeability, and $f$ is the frequency.

\section{Design of the TCA}

\subsection{Design of the mover}

The mover consists of a guiding structure and a plate. As shown in Fig. 3, the guiding structure can minimize the shaking during the motion of the mover. Furthermore, the surge current can bypass to the ground through the guiding structure. In the plate, the eddy current is generated and this produces the repulsive force. 


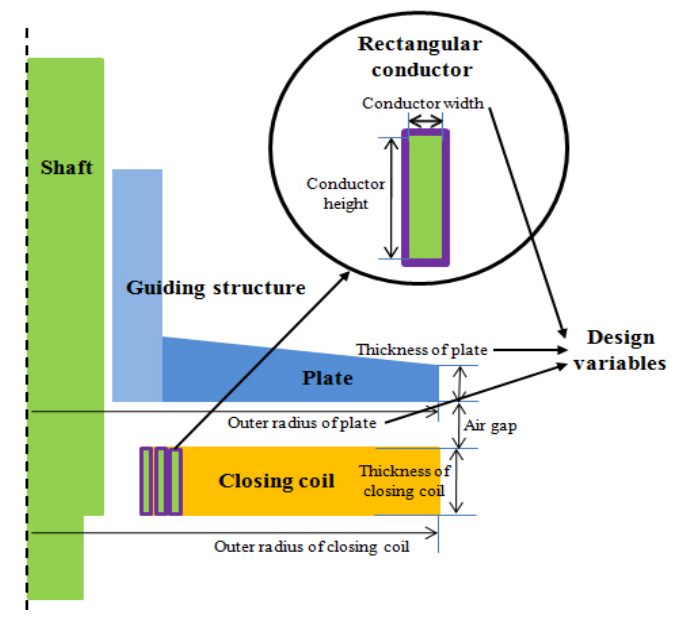

Fig. 3. The design variables of the TCA

\subsubsection{Material}

The mover should be made by a highly conductive material to generate an eddy current for a repulsive force and to make the path for the surge current into the ground. Furthermore, to increase the speed of the motion, a light material should be used for the mover.

To decrease the completion time, a different material is recommended for the plate and the guiding structure. Specifically, the copper is useful for the plate to generate the repulsive force. The aluminum is valuable for the guiding structure to reduce the weight of the mover. However, the mover is constructed by one material to increase the mechanical stability.

In a no load condition, although the conductivity of the aluminum is lower than that of the copper, the completion time of the aluminum is shorter than that of the copper because the weight is more effective compared to the conductivity in the no load condition. As the load is increased, the effect of the conductivity has more effect compared to the weight of the mover. Hence, when the aluminum is used for the mover of the TCA, the completion time is increased dramatically as the load is increased. However, when the copper is used for the mover of the TCA, the variation of the completion time is stable for the increase of the load. For this reason, the copper is chosen as the material for the mover due to its robust

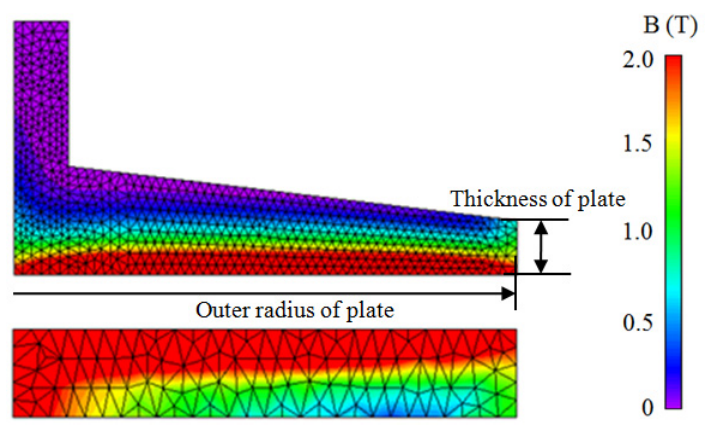

Fig. 4. The distribution of the magnetic flux density of the TCA characteristic about the load increase with the high conductivity [9].

\subsubsection{The outer radius and the thickness of the plate}

As demonstrated in Fig. 4, the variation of the flux is concentrated in the upper part of the closing coil and the lower part of the plate. The amplitude of the repulsive force is determined by the variation of the flux. Hence, the thickness and the outer radius of the plate are significant design variables [9].

\subsection{Design of the closing coil}

To complete the operation in a short time, the TCA should generate a large repulsive force in a short time. This is because the repulsive force is proportional to the variation of the magnetic flux of the conductor. The current into the conductor should be increased within a short time. For the rapid increase of the current, the resistance of the conductor should be decreased to lower the damping ratio of the conductor. To decrease the resistance of the conductor maintaining the total current in the fixed space, the rectangular conductor is used instead of the conventional round conductor in this research.

When the round conductor is used for the closing coil, the empty space between the conductors is increased as the area of the conductor expands as shown in Fig. 5(a). On the other hand, when the rectangular conductor is used for the closing coil, the space can be used efficiently without

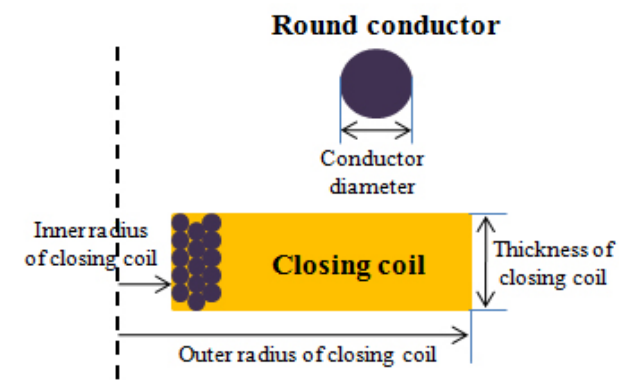

(a)

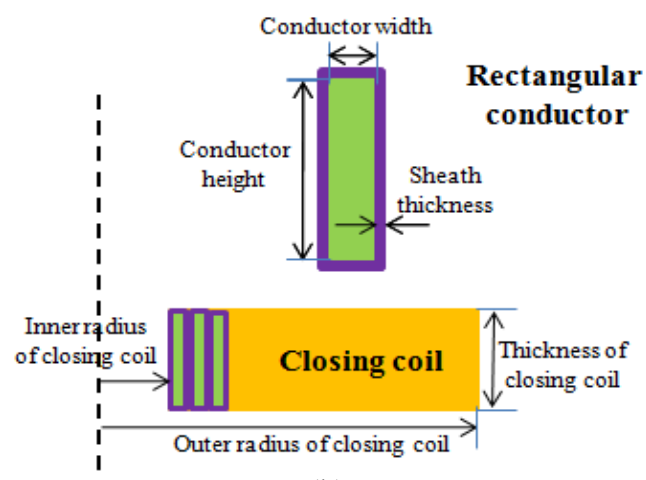

(b)

Fig. 5. The closing coil of the TCA by using: (a) The round conductor; (b) The rectangular conductor 
empty space as shown in Fig. 5(b). Hence, by using the rectangular conductor, the performance of the TCA can be increased more than when the round conductor is used because the fill factor of the rectangular conductor is larger and the resistance is smaller than that of the round conductor.

Compared with the other type of the actuators, the high current density can be applied into the TCA because of its extremely shorter operation time. Therefore, the size of the TCA can be decreased compare to the other type of actuator because higher current density can be applied into the TCA than other type of actuator.

The maximum applicable current into the closing coil is limited by the cost of the switching device of the driving system. In the same current density, higher current flows in the rectangular conductor compared to the round conductor. In other words, high current can flow in the TCA using both an extremely high current density and the rectangular conductor. Hence, the switching device should cover the high current. This causes the increase of the cost for the driving system and limits the applicable current into the closing coil of the TCA.

\section{Optimal Design of the TCA using an Optimization Algorithm}

\subsection{Optimization algorithm}

Optimization of the electric machine is finding the superior model in the aspect of the objective while satisfying the required conditions. For the optimization of the electric machine, design parameters, objective, requirements, and constraints should be determined and considered.

For the design of the TCA, the time step analysis using finite element analysis (FEA) should be carried out for the accurate analysis. However, the time step analysis using FEA requires much time consumption. Hence, when the TCA is designed optimally by using a trial-and-error method via the time step analysis using FEA, much time and effort are required.

To solve this problem, an useful optimal design method using the gradient assisted simplex method (GASM) is proposed for the optimal design of the TCA. The GASM can converge rapidly compared to the conventional simplex method [13].

Through the GASM algorithm and the time step analysis using FEA, the TCA can be designed optimally with less number of function calls and effort than the conventional trial-and-error method.

\subsection{Optimal design of the TCA using GASM}

\subsubsection{Objective and constraints for the optimization of the TCA}

The TCA as the arc eliminator has to complete the operation in a short time to eliminate the arc as soon as possible to minimize damages of the load circuit by the fault current. Hence, the completion time should be the objective for the optimization of the TCA.

The constraints for the optimization of the TCA are the maximum current of the switching device in the driver and the maximum current density of the closing coil. The maximum current, $4 \mathrm{kA}$, is decided by considering the switching device and the maximum current density $1 \mathrm{kA} /$ $\mathrm{mm}^{2}$ is determined based on the report [8].

To consider the constraint of the maximum current during the optimization, a novel method is required. Hence, a linear combination function using unit step function, which can consider the current constraint in the optimization process, is proposed as (7) in this research.

$$
F(\mathrm{x})=f(\mathrm{x})+g(\mathrm{x}) u\left\{g(\mathrm{x})-I_{\max }\right\}
$$

where $\mathrm{x}=\left[\mathrm{x}_{1}, \ldots, \mathrm{x}_{\mathrm{i}}\right]$ is the design variables, $\mathrm{x}_{\mathrm{i}}$ is $i^{\text {th }}$ design variable, $f(\mathrm{x})$ is the objective function about the completion time, $g(\mathrm{x})$ is the function about the current, $I_{\max }$ is the maximum current as the constraint, and $u\{\mathrm{~g}(\mathrm{x})\}$ is the unit step function. The $F(\mathrm{x})$ is the minimization problem since the main objective function is completion time and the shorter completion time means the better performance than the longer one. The role of the second term of the $F(\mathrm{x})$ is to limit the current under the maximum current. If the smaller current than $I_{\max }$ flows into the closing coil, the second term is zero. On the other hand, if the larger current than $I_{\max }$ flows into the closing coil, the second term has very large value. Hence, the algorithm can recognize the large current beyond the limit while minimizing the completion time only with $F(\mathrm{x})$.

To optimize the $\mathrm{N}$-dimensional problem, the simplex method uses $(\mathrm{N}+1)$ nodes [14]. Hence, the simplex method for a two-dimensional problem uses three nodes as shown in Fig. 6, in which $x_{0}, x_{1}$, and $x_{2}$ are the number of nodes and $x_{b}, x_{w}$, and $x_{s w}$ are nodes that have the best, worst, and second worst values of the objective function, respectively.

If the current is not over the constraint of the maximum current at all the position of $x_{b}, x_{w}$, and $x_{s w}$ and the reflection operator is used then the next solution will be $x_{r}$ as shown in Fig. 7(a). However, if the current at the position of $x_{0}$ over the current constraint then $x_{0}, x_{1}$, and $x_{2}$ becomes respectively $x_{w}, x_{s w}$, and $x_{b}$, by the linear

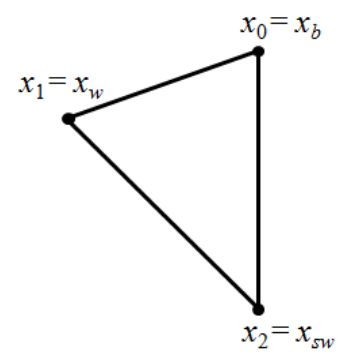

Fig. 6. Definition of the points and lines 


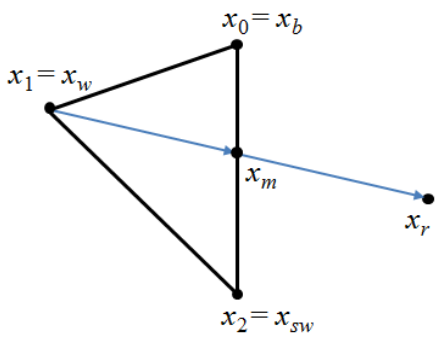

(a)

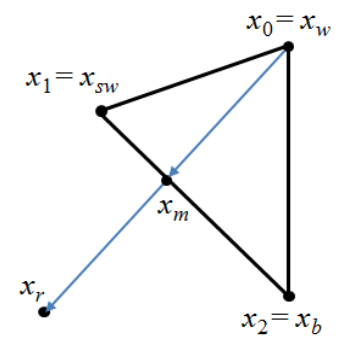

(b)
Fig. 7. The application of the proposed function (7) into the GASM optimization algorithm assuming that the next solution was found through the reflection operator: (a) when the current is not over the maximum current constraint; (b) when the current at the position of $\mathrm{x}_{0}$ over the current constraint

Table 1. Result of the optimization

\begin{tabular}{c|c|c}
\hline Design variable & Range $(\mathrm{mm})$ & Result of the optimization $(\mathrm{mm})$ \\
\hline Outer radius of the plate & $15 \sim 40$ & 40.00 \\
\hline Plate thickness & $3 \sim 5$ & 3.04 \\
\hline Conductor width & $0.5 \sim 1.5$ & 1.02 \\
\hline
\end{tabular}

combination function using the unit step function and the next solution will be $x_{r}$ as shown in Fig. 7(b).

\subsubsection{Design variables}

As demonstrated in Fig. 4, the variation of the flux is concentrated in the upper part of the closing coil and the lower part of the plate. This variation of the flux determines the amplitude of the repulsive force. Hence, the thickness of the plate, the outer radius of the plate, and the conductor width should be design variables as illustrated in Fig. 3.

The thickness and the outer radius of the plate should be large enough to cover the maximum flux minimizing the mass of the mover because the mover should move rapidly to shorten the completion time. The size of the conductor width should be determined to maximize the repulsive force in the constraint range of the current. The ranges of the design variables are tabulated in Table 1.

\subsubsection{Optimal design results}

- The outer radius of the plate

The optimal outer radius of the plate is determined as $40 \mathrm{~mm}$, which is the same length as the outer radius of the winding. The efficiency of the repulsive force per mass of the mover is maximized when the facing area between the mover and the winding is maximized because the repulsive force is generated by the variation of the flux, which is concentrated in the lower part of the plate and the upper part of the winding.

- The thickness of the plate

Because the mover can be damaged when the mover arrives at the fixed electrode with a rapid speed, the thickness of the plate should be over about $3 \mathrm{~mm}$. Furthermore, the thickness should be large enough to cover the maximum flux. However, to decrease the completion time, the mass of the mover should be minimized. Through the optimization, the optimal thickness of the plate is found as $3.04 \mathrm{~mm}$.

\section{- The conductor width}

According to the variation of the conductor width in the available space, the number of turns, resistance, and current are varied. If the conductor width is increased in the fixed space, the number of turns is decreased. In this case, the current is increased due to the resistance of the closing coil is decreased. In other words, the repulsive force, which depends on the current of the conductor, can be controlled by the variation of the conductor width.

Because the TCA operates in an extremely short time, the maximum current is limited mainly by the cost of the driving devices and the heat generation of the closing coil. The optimization result of the conductor width, which minimizes the completion time under the current limitation, is $1.02 \mathrm{~mm}$. Taking into account the manufacturing process, the final conductor width is determined to be $1 \mathrm{~mm}$.

\section{Comparison between Experimental data and Analysis results}

Based on the requirement and restriction for the TCA shown in Table 2, the TCA is designed optimally and prototyped as shown in Fig. 8, significant characteristics for the TCA, which are the current and displacement of the TCA, are analyzed by using the proposed analysis method

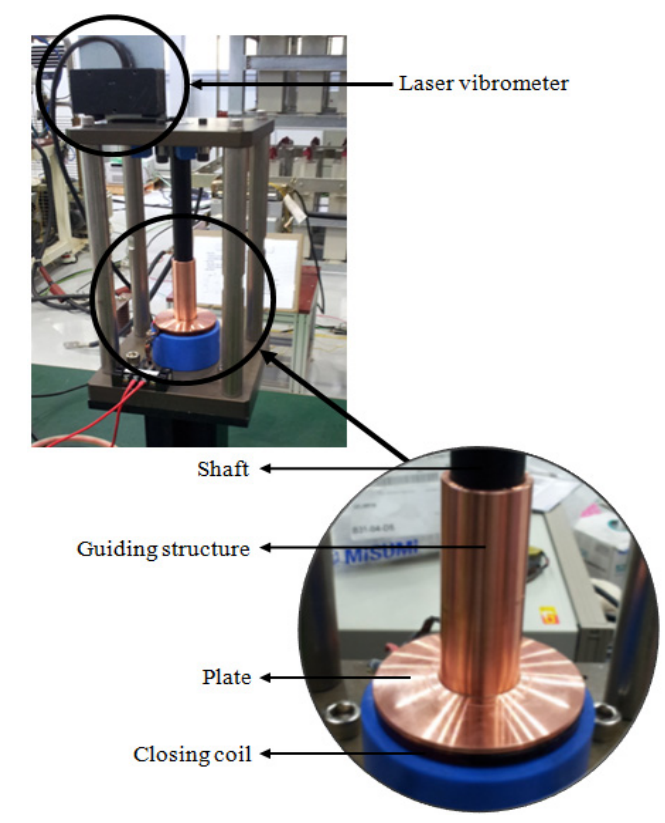

Fig. 8. Prototype of the TCA 
Table 2. Requirements and restrictions of the TCA

\begin{tabular}{c|c}
\hline Specification & Value \\
\hline Outer radius of the TCA & Less than $40 \mathrm{~mm}$ \\
\hline Completion time & Less than $3.5 \mathrm{~ms}$ \\
\hline
\end{tabular}

Table 3. Result of the optimization

\begin{tabular}{c|c|c|c}
\hline & Calculated results & Experimental results & Error \\
\hline Maximum current & $3088 \mathrm{~A}$ & $2960 \mathrm{~A}$ & $4.15 \%$ \\
\hline Completion time & $3.072 \mathrm{~ms}$ & $3.348 \mathrm{~ms}$ & $8.23 \%$ \\
\hline
\end{tabular}

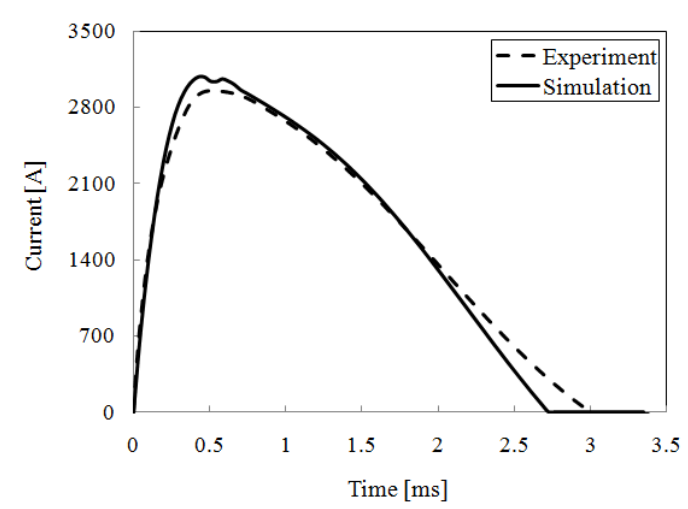

(a)

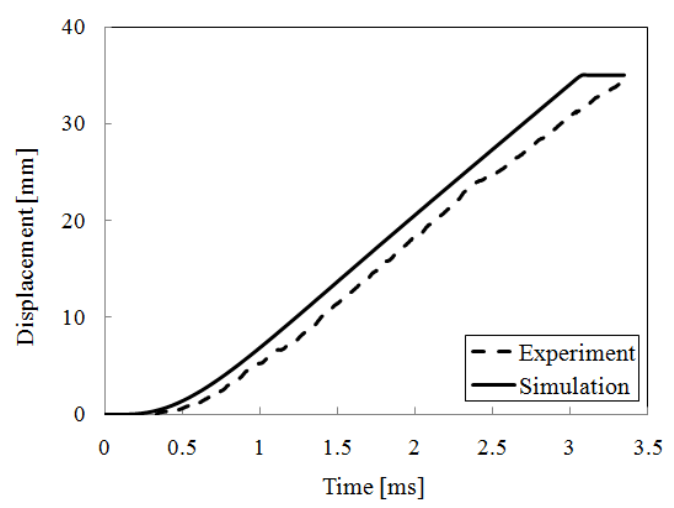

(b)

Fig. 9. Comparison between calculated data and experimental results: (a) Current of the TCA; (b) Displacement of the TCA

and compared with the experimental data, as illustrated in Fig. 9. Specific data of the significant characteristics of the TCA and its error between the experimental results and calculated data are tabulated in Table 3.

It is assumed that the error between the experimental data and calculated results is mainly caused by the ignorance of the friction between the shaft and the guiding structure in the analysis because this friction is difficult to be considered in the analysis due to its nonlinearity. However, the calculated results agree well with the experimental data within the acceptable error as shown in Fig. 9 and Table 3. Hence, it is verified that the proposed analysis method is correct.

The TCA is designed optimally by using the proposed optimal design method and its prototype satisfy with the requirements. Furthermore, by using the optimization method, time and effort for the design are mitigated compared to the conventional trial-and-error design method. Hence, it is testified that the optimization algorithm for the TCA is useful. Furthermore, via the test of the prototyped TCA, the usefulness for the arc eliminator is validated.

\section{Conclusion}

The TCA has received much attention due to its fascinating merits. Hence, the findings presented in this paper are noteworthy in the aspect that the optimal design strategy for the TCA are proposed by using the optimization algorithm and via the precise inspection of the significant variables of the TCA. The usefulness of the proposed method is verified through the experiment.

Furthermore, this research is significant in that the superiority of the TCA for the application of the arc eliminator for the high power $\mathrm{CB}$ is testified via the prototyped TCA and this result can help the proliferation of the TCA for arc eliminators.

\section{Acknowledgements}

This research was supported by the Seoul National University Electric Power Research Institute.

\section{References}

[1] S. Fang, H. Lin, S. L. Ho, X. Wang, P. Jin and H. Liu, "Characteristics analysis and simulation of permanent magnet actuator with a new control method for air circuit breaker", IEEE Trans. Magnetics, vol. 45, no. 10, pp. 4566-4569, Oct. 2009.

[2] S.Fang, H.Lin, and S.L. Ho, "Transient co-simulation of low voltage circuit breaker with permanent magnet actuator", IEEE Trans. Magnetics, vol. 45, no. 3, pp. 1242-1245, Mar. 2009.

[3] C. K. Lim, I. M. Chen, L. Yan, G. Yang, and K. M. Lee, "Electromechanical modeling of a permanentmagnet spherical actuator based on magnetic-dipolemoment principle", IEEE Trans. Industrial Elec., vol. 56, no. 5, pp. 1640-1648, May. 2009.

[4] J. Wang, W. Wang, G. W. Jewell and D. Howe, "A novel spherical actuator: design and control", IEEE Trans. Magnetics, vol.33, no.5, pp.4209-4211, Sep. 1997.

[5] R. E. Clark, G. W. Jewell, P. Stewart and D. Howe, "Tailoring force-displacement characteristics in medium-stroke linear variable reluctance actuators", IEEE Trans. Magnetics, vol. 38, no. 5, pp. 3267-3269, Sep. 2002. 
[6] T. Takeuchi, K Koyama, and M. Tsukima, "Electromagnetic analysis coupled with motion for highspeed circuit breakers of eddy current repulsion using the tableau approach", Electrical Engineering in Japan., vol. 152, no. 4, pp. 8-16, 2005.

[7] W. Li, Y. W. Jeong, H. S. Yoon, and C. S. Koh, "Analysis of parameters influence on the characteristics of thomson coil type actuator of arc eliminator using adaptive segmentation equivalent circuit method", Journal of Electrical Engineering \& Technology, vol. 5, no. 2, pp. 282-289, 2010.

[8] A. Bissal, J. Magnusson, E. Salinas, G. Engdahl, and A. Eriksson, "On the design of ultra-fast electromechanical actuators: a comprehensive multi-physical simulation model", Electromagnetic Field Problems and Applications, Jun. 2012.

[9] D. K. Lim, D. K. Woo, I. W. Kim, D. K. Shin, J. S. Ro, T. K. Chung, and H. K. Jung, "Characteristic analysis and design of a thomson coil actuator using an analytic method and a numerical method", IEEE Trans. Magnetics, vol. 36, no. 5, pp. 3571-3574, Sep. 2000.

[10] D. J. Cho, D. K. Woo, J. S. Ro, T. K. Chung, and H. K. Jung, "Novel electromagnetic actuator using a permanent magnet and an inter-locking mechanism for a magnetic switch", IEEE Trans. Mechatronics, vol. 49, no. 5, May. 2013.

[11] J. S. Rho, S. Y. Kwak, T. K. Chung, and H. K. Jung, "Analysis of a nanopositioning actuator using a numerical method combined with a analytic method", International Journal of Applied Electromagnetics and Mechanics, vol. 28, no. 3, pp. 379-394, Sep. 2008.

[12] J. S. Rho, C. H. Lee, T. K. Chung, C. H. Im, and H. $\mathrm{K}$. Jung, "Analysis of a nano positioning actuator using numerical and analytic methods", Smart Materials \& Structures, 17 025025, Feb. 2008.

[13] I. W. Kim, D. K. Woo, D. K. Lim, S. Y. Jung, C. G. Lee, J. S. Ro, and H. K. Jung, "Minimization of a cogging torque for an interior permanent magnet synchronous machine using a novel hybrid optimization algorithm", Journal of Electrical Engineering \& Technology, vol. 8, pp. 742-748, 2013.

[14] J. A. Neider, R. Mead. "A simplex method for function minimization", The Computer Journal $7 \mathrm{pp}$. 308-313, 1965.

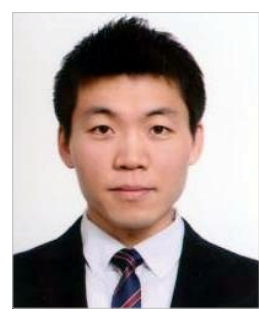

Dong-Kuk Lim He received B.S. degree in the electrical engineering from Dongguk University, Seoul, Korea, in 2010. He is currently working toward the Ph.D. degree in Electrical and Computer Engineering from the Seoul National University, Seoul, Korea. His current research interests include analysis and optimal design of electrical machines.

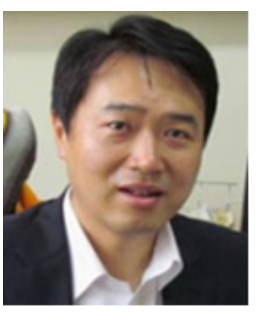

Sang-Yong Jung He received the B.S., M.S., and Ph.D. degrees in electrical engineering from Seoul National University, Seoul, Korea, in 1997, 1999, and 2003, respectively. From 2003 to 2006, he was a Senior Research Engineer with the R\&D Division, Hyundai Motor Company, Korea, and the R\&D Division, Kia Motor, Korea. He is currently an Associate Professor with the School of Electronic and Electrical Engineering, Sungkyunkwan University, Suwon, Korea. His research interests include the numerical analysis and optimal design of electric machines and power apparatus.

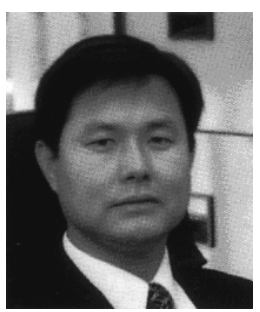

Hyun-Kyo Jung He received the B.S., M.S., and Ph.D. degree in Electrical engineering from the Seoul National University, Seoul, Korea, in 1979, 1981, and 1984, respectively. From 1985 to 1994, he was a member of the faculty with Kangwon National University. From 1987 to 1989, he was with the Polytechnic University of Brooklyn, Brooklyn, NY. From 1999 to 2000, he was a Visiting Professor with the University of California at Berkeley. He is currently a Professor at the School of Electrical and Computer Engineering, Seoul National University. His research interests are the analysis and design of the electric machine.

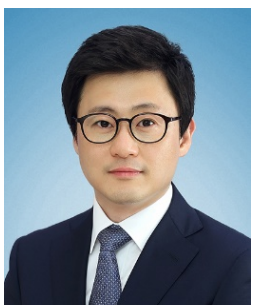

Jong-Suk Ro He earned a $\mathrm{PhD}$ in Electrical engineering from Seoul National University (SNU), Seoul, Korea, in 2008. In 2001, he received the B.S. degree in Mechanical Engineering from Han-Yang University, Seoul, Korea. Currently, he is an Assistant Professor at School of Electrical and Electronics Engineering, Chung-Ang University, Seoul, Korea. From 2013 to 2016, he was at Brain Korea 21 Plus, SNU as a BK Assistant Professor. In 2014, he was with the University of BATH, Bath, UK. He carried out research at Electrical Energy Conversion System Research Division of Korea Electrical Engineering \& Science Research Institute as a Researcher in 2013. From 2012 to 2013, he was at Brain Korea 21 Information Technology of SNU as a PostDoctoral Fellow. He conducted research at R\&D center of Samsung Electronics as a Senior Engineer from 2008 to 2012. His research interest is characteristic analysis and optimal design of electric machines and systems. 